\title{
Hydrothermal synthesis of gold nanoplates and their structure-dependent LSPR properties - CORRIGENDUM
}

Yanting Liu, Lijun Yang, and Yajing Shen

doi: https://doi.org/10.1557/jmr.2018.274, Published by Materials Research Society with Cambridge University Press, 13 August 2018.

In Liu et al., ${ }^{1}$ Lijun Yang is a co-corresponding author along with Yajin Shen. The corresponding author note should have read as follows:

Address all correspondence to these authors:

e-mail: yljtj@hit.edu.cn

e-mail: yajishen@cityu.edu

\section{REFERENCE}

1. Y. Liu, L. Yang, and Y. Shen: Hydrothermal synthesis of gold nanoplates and their structure-dependent LSPR properties. J. Mater. Res. 33, 2671 (2018). 